\title{
Synaptic balance due to homeostatically self-organized quasicritical dynamics
}

\author{
Mauricio Girardi-Schappo $\odot,{ }^{1, *}, \dagger$ Ludmila Brochini, ${ }^{2}$ Ariadne A. Costa, ${ }^{3}$ Tawan T. A. Carvalho $\odot,{ }^{4}$ and Osame Kinouchi $\odot^{1,{ }^{*}, \sharp}$ \\ ${ }^{1}$ Universidade de São Paulo, FFCLRP, Departamento de Física, Ribeirão Preto, SP, 14040-901, Brazil \\ ${ }^{2}$ Universidade de São Paulo, Instituto de Matemática e Estatística, São Paulo, SP, 05508-090, Brazil \\ ${ }^{3}$ Universidade Federal de Goiás - Regional Jataí, Unidade Acadêmica Especial de Ciências Exatas, Jataí, GO, 75801-615, Brazil \\ ${ }^{4}$ Universidade Federal de Pernambuco, Departamento de Física, Recife, PE, 50670-901, Brazil
}

(Received 30 July 2019; accepted 23 January 2020; published 20 February 2020)

\begin{abstract}
Recent experiments suggested that a homeostatic regulation of synaptic balance leads the visual system to recover and maintain a regime of power-law avalanches. Here we study an excitatory/inhibitory (E/I) meanfield neuronal network that has a critical point with power-law avalanches and synaptic balance. When shortterm depression in inhibitory synapses and firing threshold adaptation are added, the system hovers around the critical point. This homeostatically self-organized quasicritical (SOqC) dynamics generates E/I synaptic current cancellation in fast timescales, causing fluctuation-driven asynchronous-irregular (AI) firing. We present the full phase diagram of the model without adaptation varying external input versus synaptic coupling. This system has a rich dynamical repertoire of spiking patterns: synchronous regular (SR), asynchronous regular (AR), synchronous irregular (SI), slow oscillations (SO), and AI. It also presents dynamic balance of synaptic currents, since inhibitory currents try and compensate excitatory currents over time, resulting in both of them scaling linearly with external input. Our model thus unifies two different perspectives on cortical spontaneous activity: both critical avalanches and fluctuation-driven AI firing arise from SOqC homeostatic adaptation and are indeed two sides of the same coin.
\end{abstract}

DOI: 10.1103/PhysRevResearch.2.012042

Experimental and theoretical evidence suggests that spontaneous cortical activity happens in the form of asynchronous irregular firing patterns (AI). This could be generated by the balance of excitatory/inhibitory (E/I) synaptic currents entering individual neurons (see Refs. [1,2]): inhibition has to nearly compensate excitation, such that cells remain near their firing threshold and fire sporadically, generating a fluctuation-driven regime [2]. These firings may be organized in avalanches of action potentials that spread throughout the cortex. Critical avalanches are known to enable the propagation of fluctuations through local interactions due to longrange spatiotemporal correlations [3], generating optimized processing, and functional features [4-8].

Two important issues remain: (i) how to self-organize a neuronal network close to a critical point and (ii) could a network display an AI firing pattern through this selforganization? Concerning the first point, it has been shown that simple local homeostatic mechanisms, such as dynamical synapses [9-13] and dynamical neuronal gains [14-16], are sufficient to drive networks towards the so-called selforganized quasicritical state (SOqC as defined by Bonachela and Muñoz [10,17]). Particularly, our model requires two

\footnotetext{
${ }^{*}$ These authors contributed equally to this work.

†girardi.s@gmail.com

†osame@ ffclrp.usp.br
}

Published by the American Physical Society under the terms of the Creative Commons Attribution 4.0 International license. Further distribution of this work must maintain attribution to the author(s) and the published article's title, journal citation, and DOI. independent homeostatic mechanisms to generate the $\mathrm{SOqC}$ dynamics: plasticity in the inhibitory synapses [18] and adaptive firing thresholds [19].

As for the second point, we will show that our homeostatic mechanisms for $\mathrm{SOqC}$ generate a near cancellation of excitatory/inhibitory (E/I) synaptic currents that produces a fluctuation-driven AI regime. Therefore AI is a direct consequence of the hovering around a critical point where the system displays quasicritical power-law avalanches. Indeed, recent experiments show homeostatic regulation of network activity close to a critical state happening most probably through the adaptation of inhibitory synapses [20].

There have been attempts to model E/I networks in the context of criticality [21-24]. However, none of these models have shown that neuronal avalanches with the correct exponents arise when E/I synaptic currents cancel each other. Also, none of these models show that synaptic currents balance each other in the vicinity of a critical point. Not only the $\mathrm{SOqC}$ dynamics proposed here does that, but it also generates activity where avalanches and AI spiking coexist.

Without $\mathrm{SOqC}$, we have a static system presenting the typical synchronicity states of E/I networks exemplified by Brunel's model [25]: synchronous regular (SR), asynchronous regular (AR), synchronous irregular (SI), and asynchronous irregular (AI). This system has a directed percolation (DP) critical point with power-law avalanches, and dynamic balance of E/I currents, since inhibitory inputs follow excitatory ones over time. Even though the E/I neuron ratio is $80 \%: 20 \%$ from cortical data [26], our model predicts that the ratio of coupling strengths of inhibitory to excitatory synapses does not need to be $4: 1$ to achieve the critical balanced state. 
We first define both the static and the adaptive versions of the model. Then, we make a mean-field calculation obtaining the critical exponents and phase diagrams, and discuss the dynamic states of the static network. Finally, we add SOqC homeostatic adaptation and observe the hovering around the critical balanced point that displays near cancellation of E/I currents and fluctuation-driven AI activity.

The model. We use discrete-time stochastic integrate-andfire neurons $[14,27,28]$. A Boolean variable denotes if a neuron fires $(X[t]=1)$ or not $(X[t]=0)$ at time $t$. The membrane potentials of neurons in $E$ and $I$ populations evolve as

$$
\begin{aligned}
V_{i}^{E}[t+1]= & {\left[\mu V_{i}^{E}[t]+I_{i}^{(E)}[t]+\frac{1}{N} \sum_{j=1}^{N_{E}} W_{i j}^{E E} X_{j}^{E}[t]\right.} \\
& \left.-\frac{1}{N} \sum_{j=1}^{N_{I}} W_{i j}^{E I} X_{j}^{I}[t]\right]\left(1-X_{i}^{E}[t]\right), \\
V_{i}^{I}[t+1]= & {\left[\mu V_{i}^{I}[t]+I_{i}^{(I)}[t]+\frac{1}{N} \sum_{j=1}^{N_{E}} W_{i j}^{I E} X_{j}^{E}[t]\right.} \\
& \left.-\frac{1}{N} \sum_{j=1}^{N_{I}} W_{i j}^{I I} X_{j}^{I}[t]\right]\left(1-X_{i}^{I}[t]\right),
\end{aligned}
$$

where $N=N_{E}+N_{I}$ is the total number of neurons, $\mu$ is a leakage parameter and $I_{i}^{(E) /(I)}[t]$ are external inputs over $E$ and $I$ populations, respectively. The second index in $W_{i j}^{a b}$, with $a, b \in\{E, I\}$, refers always to the presynaptic neuron. All the $W$ 's are positive (inhibition is explicitly given by the minus). The term $\left(1-X_{i}[t]\right)$ resets the voltage to zero after a spike, resulting in one time step of refractoriness. Our network is fully connected with $K=N-1$ neighbors.

The individual neurons fire following a piecewise linear probability function [see Fig. 1(a)]:

$$
\begin{aligned}
& P(X=1 \mid V) \equiv \Phi(V) \\
& \quad=(V-\theta) \Gamma \Theta(V-\theta) \Theta\left(V_{S}-V\right)+\Theta\left(V-V_{S}\right),
\end{aligned}
$$

where $\Gamma$ is the neuronal firing gain parameter, $\theta$ is a firing threshold, $V_{S}=\theta+1 / \Gamma$ is the saturation potential and $\Theta(x)$ is the Heaviside function. The firing probability $\Phi(V)$ captures the effects of membrane noises, inducing stochastic spiking. The limit $\Gamma \rightarrow \infty$ reduces to the leaky integrate-andfire (LIF) neuron with hard threshold $V_{S}=\theta$.

Order and control parameters. We assume that the synaptic weights have finite variance (are self-averaging), approximating them by their mean values $W^{a b}=\left\langle W_{i j}^{a b}\right\rangle$ (for all the $a, b \in\{E, I\}$ ). We also define the firing densities (the fraction of active sites) $\rho_{E}[t]=1 / N_{E} \sum_{j} X_{j}^{E}[t]$ and $\rho_{I}[t]=$ $1 / N_{I} \sum_{j} X_{j}^{I}[t]$. The fractions of excitatory and inhibitory neurons are $p=N_{E} / N$ and $q=1-p=N_{I} / N$, respectively. Finally, we consider only the case with a stationary average external input $I=\left\langle I_{i}[t]\right\rangle$ with finite variance over both populations.

We introduce the synaptic balance parameter $g$ by letting the synaptic weights obey $W^{E E}=W^{I E}=J$, and $W^{I I}=$ $W^{E I}=g J$ (Brunel's model A [25]). This is not a necessary assumption, but it reduces Eqs. (1) and (2) to a single iterative
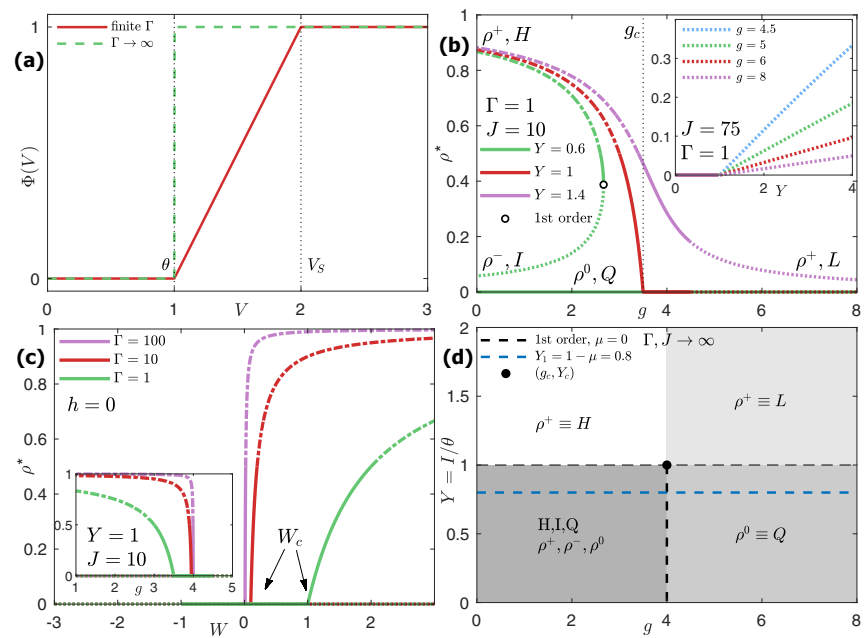

FIG. 1. Firing rate function and phase transitions. (a) Solid: soft firing threshold $(\Gamma=1)$, dashed: hard threshold $(\Gamma \rightarrow \infty)$. (b) Order parameter vs $g$ (inset: vs $Y$ ), highlighting the activity states high $(\mathrm{H})$, low (L), intermediary (I, unstable, from a fold bifurcation), and quiescent $\left(\mathrm{Q} \equiv \rho^{0}=0\right)$. (c) Order parameter $\rho^{*}$ vs $W$ for $h=0$ (inset: vs $g$ for $Y=1$ ); notice as the critical point shifts away from $W_{c}=0\left(g_{c}=4\right)$ as $\Gamma$ decreases $\left(W_{c}=1 / \Gamma\right)$. [(b) and (c)] Dot-dashed lines are marginally stable cycle- 2 attractors (SR state). Dotted lines in the $\rho^{+}(\mathrm{L})$ branch are cyclic attractors of the network (quasicycle-2 SI states). Density $\rho$ is given by Eqs. (11) and (14). (d) Phase diagram in the balanced notation $(g, Y)$ plane for the hard threshold neurons. The critical point lies at $\left(W_{c}=0, h_{c}=0\right)$ or $\left(g_{c}=4, Y_{c}=1\right)$ [bullet, Eq. (13)]. The Q phase loses stability at the horizontal dashed line $Y_{c}=1-\mu$ (or $\left.h_{c}=0\right) ; \mu=0$ (black and thin dashed line) and $\mu=0.2$ (blue dashed line). This diagram should be compared to Fig. 1 A of Ref. [25].

map that is equal for both $E / I$ populations:

$$
V_{i}[t+1]=\left[\mu V_{i}[t]+I+p J \rho_{E}[t]-q g J \rho_{I}[t]\right]\left(1-X_{i}[t]\right),
$$

where we may omit the $E / I$ superscripts. Letting the excitatory synaptic current be ${ }^{1} I^{E}[t]=p J \rho_{E}[t]$ and the inhibitory $\mathrm{be}^{2} I^{I}[t]=-q g J \rho_{I}[t]$, we define the average net synaptic current,

$$
\Delta I^{E / I}=I^{E}+I^{I}=p J \rho_{E}-q g J \rho_{I}=W \rho,
$$

where we used $\rho_{E}=\rho_{I}=\rho$ (due to the constraints added to the synaptic weights) and defined $W=(p-q g) J$ as our first control parameter. This holds because, after a neuron spikes, the voltage reset erases initial conditions and the voltages for both $E / I$ populations evolve following Eq. (4). The firing density $\rho$ is our order parameter, equivalent to the network firing frequency $v_{0}$ of Brunel's model [25].

Consider the stationary state $(1-\mu) V^{*}=I$ for $\rho=0$ in Eq. (4). When $V^{*}=\theta, \Phi\left(V^{*}\right)=0$, so we have $\rho>0$

\footnotetext{
${ }^{1}$ Not to be confused with external input over the excitatory population $I_{i}^{(E)}[t]$ in Eq. (1).

${ }^{2}$ Not to be confused with external input over the inhibitory population $I_{i}^{(I)}[t]$ in Eq. (2).
} 
for $I>(1-\mu) \theta$. Thus we define the external field $h=I-$ $\theta(1-\mu)$ as the average suprathreshold external current. The $h$ variable is our second control parameter. The parameters $(W, h)$ are usual for Statistical physics. By introducing the external current ratio, $Y=I / \theta$, we may switch from $(W, h)$ to describe the system in the balanced notation $(g, Y)$ by using $g=p / q-W /(q J)$ and $Y=(h / \theta)+1-\mu$.

Homeostatic mechanisms. To obtain a quasicritical balanced state without fine tuning, we introduce two independent homeostatic biological mechanisms: inhibition depression [20] and firing threshold adaptation [29]. We use the Levina-Hermann-Geisel short-term plasticity for the synaptic weights [9]:

$$
\begin{aligned}
W_{i j}^{I I / E I}[t+1]= & W_{i j}^{I I / E I}[t]+\frac{1}{\tau_{W}}\left(A-W_{i j}^{I I / E I}[t]\right) \\
& -u_{W} W_{i j}^{I I / E I}[t] X_{j}^{I}[t],
\end{aligned}
$$

where $\tau_{W}$ is a (large) recovery time, $A$ is the synaptic baseline and $u_{W}$ is the fraction of the synaptic strength depressed when a presynaptic neuron fires. This dynamic generates homeostatic tuning because $g$ is then $g[t]=\left\langle W_{i j}^{E I / I I}[t]\right\rangle / J$ in Eq. (4). The $\langle$.$\rangle bracket is an average over neurons i$ and $j$.

To self-organize towards zero-field $h_{c}=I-(1-\mu) \theta=$ 0 or $Y_{c}=I / \theta=1-\mu$, we add threshold adaptation:

$$
\theta_{i}[t+1]=\theta_{i}[t]-\frac{1}{\tau_{\theta}} \theta_{i}[t]+u_{\theta} \theta_{i}[t] X_{i}[t],
$$

where the parameter $u_{\theta}$ is the fractional increase in the neuron threshold after it fires, and $\tau_{\theta}$ is a recovery timescale. This dynamic is inspired by the biological mechanism of firing rate adaptation [29]. It enters the model through Eq. (3), changing $\theta$ to $\theta[t]=\left\langle\theta_{i}[t]\right\rangle$.

Mean-field calculations. We consider only the $\mu=0$, since $\mu>0$ does not present any new phenomenology (although it admits numerical solutions and analytic approximations close the critical point $[14,15])$. For this case, the stationary voltage distribution has only two delta peaks, $P_{t}(V)=\rho[t] \delta(V)+$ $(1-\rho[t]) \delta(V-V[t])$, and the number of active sites is the average of $\Phi(V)$ over $V[14,16]$,

$$
\rho[t+1]=\int \Phi(V) P_{t}(V) d V,
$$

with $V[t]$ given by Eq. (4), resulting in

$$
\rho[t+1]=(1-\rho[t]) \Gamma(W \rho[t]+h) \Theta(W \rho[t]+h) .
$$

This map has, in principle, three fixed points. For $h \leqslant 0$, there is a quiescent solution $\rho^{0}=0$ (also called the $\mathrm{Q}$ state) since the Heaviside $\Theta(x)$ function is zero in the right-hand side in Eq. (9).

The active states are the two other fixed points of the firing density (9), given by

$$
\Gamma W \rho^{2}+(1+\Gamma h-\Gamma W) \rho-\Gamma h=0,
$$

with solutions

$$
\rho^{ \pm}=\frac{\Gamma W-\Gamma h-1}{2 \Gamma W} \pm \frac{\sqrt{(\Gamma W-\Gamma h-1)^{2}+4 \Gamma^{2} W h}}{2 \Gamma W} .
$$

For $h>0(Y>1)$, there is a single solution $\rho^{+}$(corresponding to high activity $\mathrm{H}$ and low activity $\mathrm{L}$ ) because $\rho^{-}<0$. For $h<0(Y<1)$, we have a positive but unstable branch $\rho^{-}$(the intermediary solution I) that separates the stable branch $\rho^{+}$ (H) from the absorbing state $\rho^{0}(\mathrm{Q})$, see Fig. 1(b). When $h=0$ $(Y=1)$, the unstable branch vanishes into a critical point with $W=W_{c}=1 / \Gamma\left[g=g_{c}\right.$, Eq. (13) $]$.

Critical exponents. For zero-field, Eq. (10) yields $\rho^{0}=0$ (the absorbing quiescent phase, $\mathrm{Q}$ ), stable for $W<W_{c} \equiv 1 / \Gamma$ and an active state:

$$
\rho^{*}=\frac{\Gamma W-1}{\Gamma W}=\frac{W-W_{c}}{W} \sim\left(W-W_{c}\right)^{\beta},
$$

with $\beta=1$, stable for $W>W_{c}=1 / \Gamma$. The field exponent is obtained by isolating $h$ from Eq. (10) and expanding for small $\rho$ (due to small external $h$ ) with $W=W_{c}$, resulting in $\rho^{*} \sim$ $\left(h / W_{c}\right)^{1 / \delta_{h}}$ with $\delta_{h}=2$. The exponent of the susceptibility, $\chi=\partial \rho / \partial h \sim\left|W-W_{c}\right|^{-\gamma}$, using $\Gamma=1 / W_{c}$, is $\gamma=1$.

These exponents pertain to the mean-field directed percolation (DP) universality class [30-32], the framework that has been proposed to describe neuronal avalanches [33,34]. The variance of the network activity is $\operatorname{Var}(\rho) \sim\left|W-W_{c}\right|^{-\gamma^{\prime}}$ with $\gamma^{\prime}=0$ [30]. This explains the jump in the coefficient of variation of $\rho$ observed by Brunel [25].

In the balanced notation, $h=h_{c}=0$ is the same as $Y_{c}=$ $\left(h_{c} / \theta\right)+1-\mu=1$ (recalling that $\mu=0$ and $\theta=1$ ). The equivalent of $W_{c}=1 / \Gamma$ is given by

$$
g_{c}=\frac{p}{q}-\frac{W_{c}}{q J}=4-\frac{5}{\Gamma J},
$$

where the usual cortical estimates $p=80 \%$ and $q=20 \%$ were used [26]. This generalizes the usual condition $g_{c} \approx 4$ : if neurons have a soft threshold (finite $\Gamma$ ) or the synapses are weak (finite $J$ ), the critical balance point shifts towards lower values of $g$ [Fig. 1(c)]. The phase diagram for large $\Gamma J$ (i.e., hard threshold LIF neurons) is shown in Fig. 1(d) and matches exactly the one obtained by Brunel [25].

Synaptic currents of the static model. We can write Eq. (11) in the balanced notation by letting $h=(Y-1) \theta$ and $W=$ $(p-q g) J$ [see Fig. 1(b)]:

$$
\begin{aligned}
\rho^{ \pm}= & \frac{1}{2}+\frac{\rho_{1}}{2 \Gamma \theta(Y-1)}+\frac{\rho_{1}}{2} \\
& \pm \sqrt{\left(\frac{1}{2}+\frac{\rho_{1}}{2 \Gamma \theta(Y-1)}+\frac{\rho_{1}}{2}\right)^{2}-\rho_{1}},
\end{aligned}
$$

where $\rho_{1}=(Y-1) \theta /[J q(g-p / q)]$ is the first-order expansion of Eq. (14).

The synaptic currents are balanced if the net synaptic current from Eq. (4) is zero, $\Delta I^{E / I}=W \rho=0$, such that either $W=(p-q g) J=0$ (i.e., $g=g_{\text {bal }}=p / q$ for $\left.Y>1\right)$, or $\rho=0$ (i.e., the quiescent solution of the subcritical and critical states, $g \geqslant g_{c}$ and $\left.Y \leqslant 1\right)$. For $g \neq p / q$, the synaptic currents scale linearly with the external input. We can see that by expanding Eq. (14) for small $Y$, giving $\rho \approx \rho_{1}$ :

$$
\begin{gathered}
I^{E}=p J \rho_{1}=\frac{p / q}{g-p / q}(Y-1), \\
I^{I}=-q g J \rho_{1}=-\frac{g}{g-p / q}(Y-1) .
\end{gathered}
$$



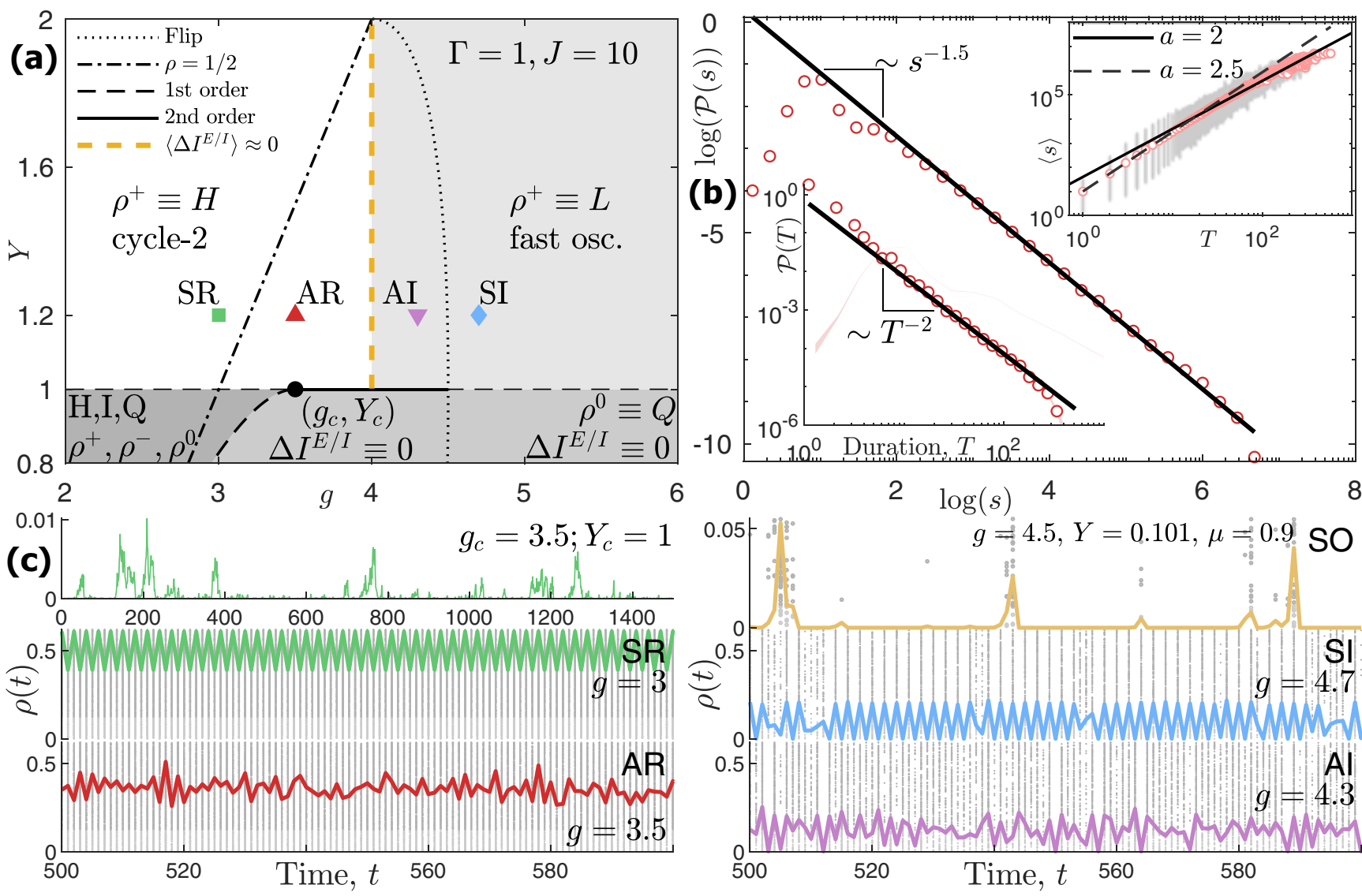

FIG. 2. Avalanches and firing patterns. (a) Phase diagram for $\mu=0$, and $\Gamma J=10$; a critical line starts at $g_{c}=3.5$, see Eq. (13), for $Y=1$. The critical point, the subcritical region with $g>g_{\text {Fold }} ; \leqslant 1$, and the supercritical region $g=4 ; Y>1$ have balanced synaptic currents, such that the net current is $\Delta I^{E / I}=I^{E}+I^{I} \approx 0$. At $Y=1.2$, from left to right: SR/cycle-2 $(g=3), \mathrm{AR} / \mathrm{High}(g=3.5), \mathrm{AI} / \mathrm{Low}(g=4.3)$, and $\mathrm{SI} /$ fast oscillations $(g=4.7)$. SR and AR are separated by a bifurcation tho cycle-2 due to the refractory period; SI and AI are separated by a flip bifurcation. (b) Distribution of avalanche sizes (main plot, $\tau=1.5$ ) and duration (bottom inset, $\tau_{t}=2$ ) at the critical point. Top inset: size and duration scaling law $\langle s\rangle \sim T^{a}$ has a crossover with $a=2.5$ for small avalanches (a finite-size effect) and $a=2$ for the rest of the data. (c) Network simulation results ( $N=10^{6}$ neurons), $\rho[t]$, for the points in (a). From the top left to the bottom right panel: critical point absorbing-state avalanches (peaks); SR, AR, SO (slow waves for $Y \gtrsim Y_{c}=1-\mu, \mu=0.9, Y=0.101$ ), SI, and AI. The background shows the raster plot of 1,000 randomly selected neurons.

The variable $\rho \approx \rho_{1}=I^{E} /(p J)$ is shown in the inset of Fig. 1(b). These currents saturate for large enough $\Gamma J$. This linear scaling highlights the dynamic balance of synaptic inputs, as inhibition tracks excitation over time [25,35].

Phase diagram. The soft threshold neurons' phase diagram is shown in Fig. 2(a). The curves are bifurcations of the stable fixed point $\rho^{+}$in Eq. (14): (i) a fold bifurcation, i.e., a firstorder phase transition for $Y<1$ that ends in the critical point $\left(g_{c}, Y_{c}\right)$. (ii) a bifurcation to cycle-2 that separates SR from AR when $\rho^{+}=1 / 2$, because the refractory period does not allow a stable fixed point with $\rho^{+}>1 / 2$, generating bursts of synchronized activity with period $2 \mathrm{~ms}$. (iii) a flip bifurcation at $g_{\text {Flip }}=p / q+1 /(q \Gamma J)$ that separates the uniform AI from the oscillatory SI in the low activity regime. (iv) the line $Y_{c}=1$ is a continuous transcritical bifurcation for $g>g_{c}$ and $g<g_{\text {Flip }}$; and a synchronization phase transition for $g>g_{\text {Flip }}$ [Fig. 1(c), inset].

The critical balanced point at $\left(g_{c}, Y_{c}\right)$ displays powerlaw distributed avalanches with exponents $\tau=1.5$ and $\tau_{t}=$ 2 for size and duration, respectively, see Fig. 2(b). The avalanches also respect the scaling law $1 /(\sigma v z)=\left(\tau_{t}\right.$ 1)/( $\tau-1)$ [inset in Fig. 2(b)], as expected for the DP universality class [30,36,37], and observed in experiments [38].

The simulated network activity in all the six dynamical regimes is shown in Fig. 2(c). The critical point $\left(g_{c}=\right.$ $3.5, Y=1)$ displays avalanches sparked by a vanishing external stimulus. The self-sustained activity regime $\left(g<g_{c}\right)$, when summed up to an external current $Y>1$, generates the regular microscopic behaviors, SR or AR. The SR state is a marginally stable cycle- 2 of the firing density and the AR is a state of high and homogeneous activity [the $\rho^{+}$in Eq. (11)]. The addition of an external current to the inhibition dominated quiescent regime results in the low irregular activity AI (and SI if $g>g_{\text {Flip }}$ ). Slow oscillations (SO) are observed when $Y \gtrsim Y_{c}=1-\mu$ and $g \geqslant g_{c}$ for $\mu \geqslant 0$.

Homeostatic SOqC dynamics. The dynamics in the inhibitory weights tunes the system along the $g$ axis of the phase diagram. Threshold adaptation regulates the system along the $Y$ axis. Both mechanisms contribute to self-organize the network towards the critical point. For the parameters considered 


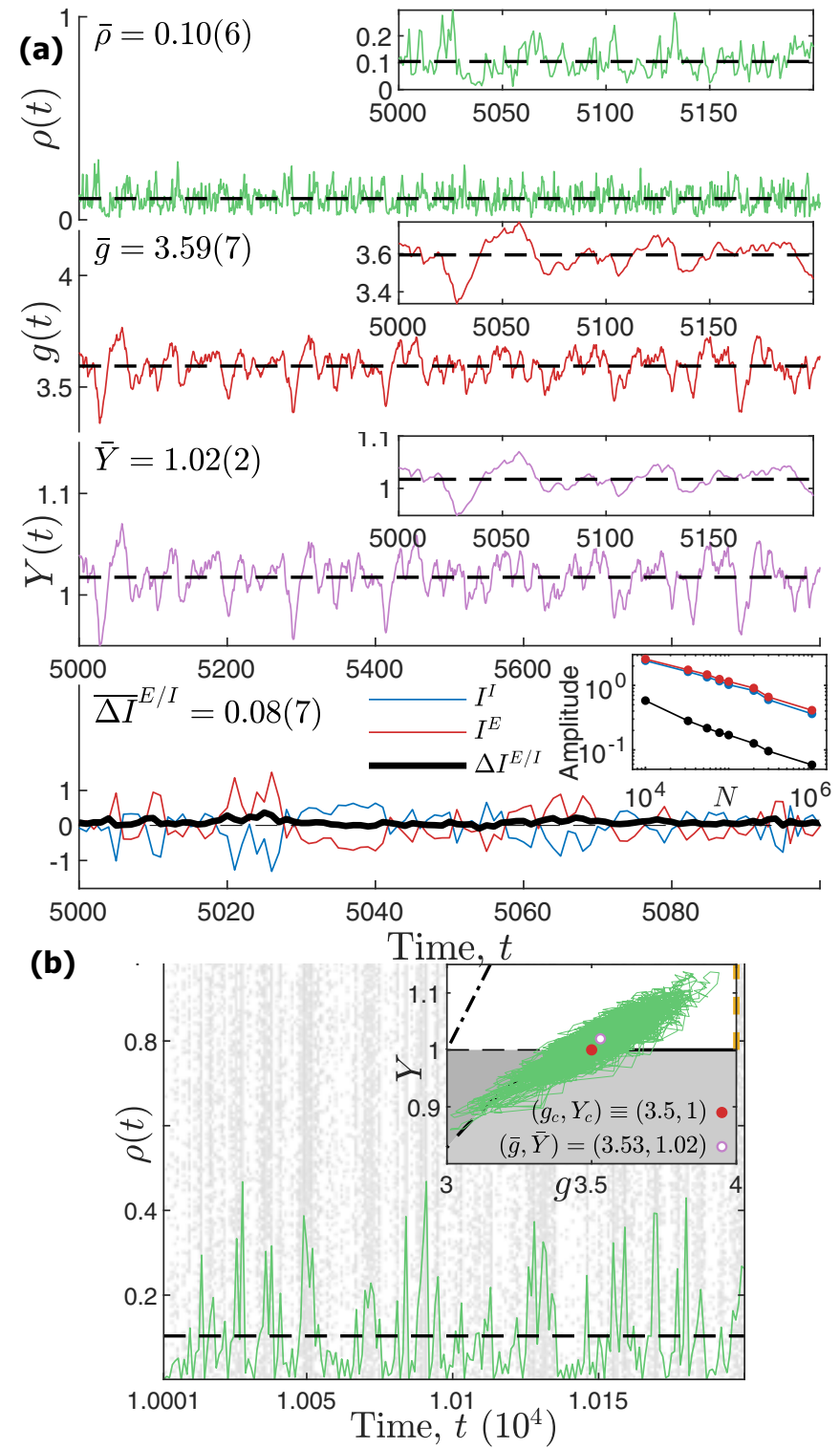

FIG. 3. Self-organization towards the balanced critical point. Parameters: $\tau_{W}=\tau_{\theta}=100, A=73.5, u_{W}=u_{\theta}=0.1, \Gamma=1$ and $J=10$. (a) Time series for $\rho[t], g[t]=W^{I I / E I}[t] / J, Y[t]=I / \theta[t]$, and the synaptic currents with $\overline{\Delta I}{ }^{E / I}=0.08(7) . I^{E}$ and $I^{I}$ have been displaced by their means. The amplitude of $I^{E}$ and $I^{I}$ are one order of magnitude larger than $\Delta I^{E / I}$ for all $N$ (bottom inset). (b) Detail of the SOqC $\rho[t]$ dynamics with a raster plot of 1000 randomly selected neurons displaying AI-like activity. (Inset) Self-organization trajectories in the $g$ vs $Y$ plane. The system hovers around the critical balanced point of the static model, $g_{c}=3.5[\bar{g}=3.59(7)]$ and $Y_{c}=1$ $[\bar{Y}=1.02(2)]$, which displays power-law avalanches.

in Fig. 2 a, the critical point is $g_{c}=3.5$ and $Y_{c}=1$, and the two independent dynamics yield $\bar{g}=\overline{\left\langle g_{i j}[t]\right\rangle}=3.59(7)$ and $\bar{Y}=\overline{\left\langle Y_{i}[t]\right\rangle}=1.02(2)$ [Fig. 3(a)].

This homeostatic tuning, however, is not perfect, since stochastic oscillations make the system hover around the critical point - a distinctive feature of self-organized quasicriticality or SOqC [10,17]—see Fig. 3(b) inset. This oscillation is triggered by finite-size (demographic) noise and its amplitude

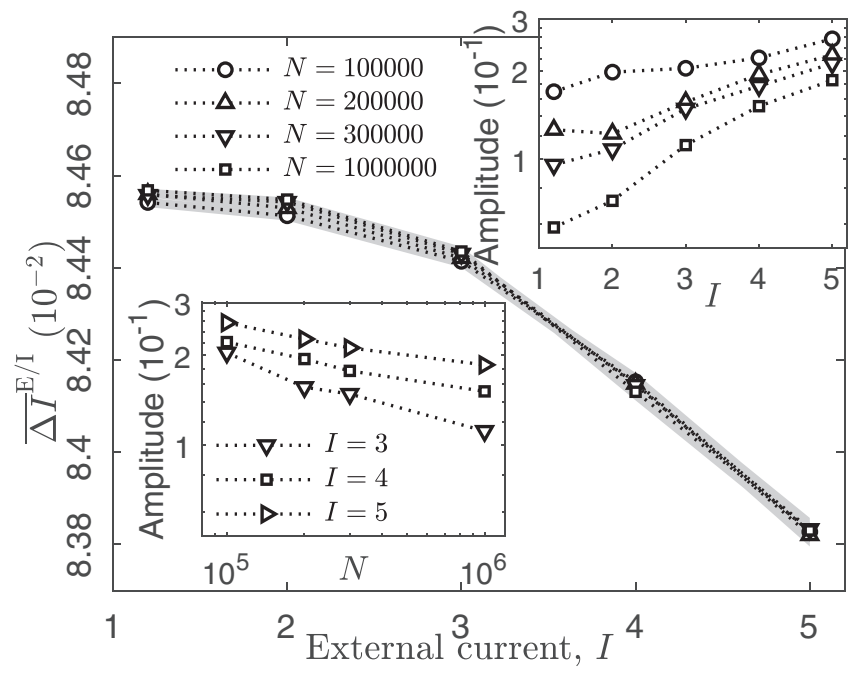

FIG. 4. Synaptic balance in SOqC. Net synaptic current $\overline{\Delta I}^{E / I}$ as function of external input $I$. The mean net synaptic current decreases with increasing $I$, making the network more balanced. Insets: Amplitude of the fluctuations of $\Delta I^{E / I}$ for different intensities of the external input (top) and for increasing network size (bottom). The decrease of the amplitude with increasing $N$ shows that the fluctuations in $\Delta I^{E / I}$ are due to finite-size effects.

decreases with increasing $N$ [inset in the bottom panel of Fig. 3(a) and bottom inset of Fig. 4]. Thus the larger the network, the closer the system gets to the critical point [16].

The spiking pattern of the SOqC dynamics is very similar to standard AI activity [compare Fig. 3(b) with Fig. 2(c)]. This happens because the E/I synaptic currents [defined in Eq. (5)] cancel each other in fast timescales, generating a net current $\Delta I^{E / I}$ that is always one order of magnitude smaller than either $I^{E}$ or $I^{I}$ [bottom panel in Fig. 3(a)].

Contrary to the static version of the model, increasing the external input $I$ on the homeostatic system slightly decreases the average net current, but increases the fluctuations of $\Delta I^{E / I}$ (see Fig. 4): the network gets more balanced and more noisy at the same time. On the other hand, independently of $I$, the fluctuations of the net synaptic current decrease with $N$ due to finite-size effects.

The nearly total cancellation of E/I currents generates sporadic fluctuations of activity the spread throughout the network (the avalanches) in an AI fashion. These avalanches should converge to nearly perfect power-law distributions for large enough $\tau_{W}=\tau_{\theta}$ [15]. Such stochastic oscillations should have low amplitude [16], but rare large events (dragonkings) also occur [15]. Although the demographic noise vanishes in the thermodynamic limit, other sources of biological noise (not included in the model and that does not vanish for large $N$ ) will continue to trigger the stochastic oscillations and the AI behavior.

Discussion. In contrast to our model, Brunel [25] used a random network, deterministic LIF neurons, noisy inputs and a distribution of delays in the synapses. In our model, noise is captured by the intrinsic stochasticity of the neurons. Our model does not have a distribution of synaptic delays, but its discrete time step implies that spikes are transmitted with a fixed delay of $1 \mathrm{~ms}$. Also, since $\Phi(0)=0$, the reset of voltages 
after spiking implements a refractory period of $1 \mathrm{~ms}$. The other ingredients do not seem to be crucial to obtain either the synchronicity/activity states or the critical balanced point.

Our mean-field calculation is valid for fully connected networks where the number of neighbors is $K=N-1$. When there is no threshold $\theta$ nor external current $I$, the condition $W=(p-q g) J$ allows our model to be directly mapped on the Kinouchi et al. [16] model. In turn, the authors showed that the latter model presents exactly the same dynamics as the sparse random network of probabilistic cellular automata where $K=\mathcal{O}(1)$, both in the static version $[5,39]$ and in the homeostatic version [12]. All these models share the meanfield DP results obtained here [14]. Calculations for the case $K=\mathcal{O}(\sqrt{N})$, as studied in [35,40], should be done to check the performance of the homeostatic mechanisms.

Heavy-tailed synaptic distributions are also expected to generate a critical point for threshold neurons [41]. Our meanfield calculations do not apply directly in this context, but our homeostatic mechanisms could still be employed to synaptic weights and thresholds to check whether the critical point would also become an attractor of that model.

While inhibition frequently increases together with excitation after the stimulation of a neuron, the reverse does not seem to happen; that is, excitation does not compensate for inhibition when the neuron is suppressed $[1,18,20]$. This suggests a self-organizing homeostatic mechanism regulating the inhibitory synapses, which was suggested to be necessary to re-establish power-law neuronal avalanches in rats [20].

This fact motivated the addition of adaptation to our model. We showed that two homeostatic mechanisms are sufficient to take the network towards any critical balance point. Adding homeostasis, we avoided fine tuning of the $g$ and $Y$ param- eters towards $g_{c}$ and $Y_{c}$. However, that comes at the cost of introducing five new parameters $\left(A, \tau_{W}, u_{W}, \tau_{\theta}, u_{\theta}\right)$ that perhaps should also be fine tuned. This is not the case: the dependence on these parameters is weak, representing a kind of gross tuning $[12,15]$. Also, if necessary, metaplasticity in longer timescales can be employed to tune these homeostatic parameters [11].

Concluding remarks. Homeostatic adaptation in synapses and firing thresholds are sufficient mechanisms to selforganize a neuronal network towards its DP critical (and synaptically balanced) point. The hovering around this attractor is due to small fluctuations in the net synaptic current, such that there is always some residual excitation driving the network activity (a sort of fluctuation-driven AI regime due to $\mathrm{SOqC}$ ). The underlying critical point shows power-law avalanches with exponents compatible with in vitro experiments [38]. Our model thus unifies two different perspectives on the spontaneous activity of the brain: power-law neuronal avalanches and fluctuation driven asynchronous-irregular firing patterns are indeed two sides of the same coin.

Acknowledgments. We thank A. C. Roque, M. Copelli and J. Stolfi for discussions. This Rapid Communication was produced as part of the FAPESP Research, Innovation and Dissemination Center for Neuromathematics (Grant No. 2013/07699-0, S. Paulo Research Foundation). L.B. thanks FAPESP (Grant No. 2016/24676-1), A.A.C. thanks FAPESP (Grants No. 2016/00430-3 and No. 2016/20945-8) and M.G.S. thanks FAPESP (Grant No. 2018/09150-9). O.K. thanks the Center for Natural and Artificial Information Processing Systems (CNAIPS)-USP and FAPESP BPE Grant No. 2019/12746-3. The present work was also realized with the support of CNPq, Conselho Nacional de Desenvolvimento Científico e Tecnológico, Brazil.
[1] S. Denève and C. K. Machens, Efficient codes and balanced networks, Nat. Neurosci. 19, 375 (2016).

[2] Y. Ahmadian and K. D. Miller, What is the dynamical regime of cerebral cortex? arXiv:1908.10101v2 [q-bio.NC].

[3] G. Ódor, Universality classes in nonequilibrium lattice systems, Rev. Mod. Phys. 76, 663 (2004).

[4] C. Haldeman and J. M. Beggs, Critical Branching Captures Activity in Living Neural Networks and Maximizes the Number of Metastable States, Phys. Rev. Lett. 94, 058101 (2005).

[5] O. Kinouchi and M. Copelli, Optimal dynamical range of excitable networks at criticality, Nat. Phys. 2, 348 (2006).

[6] W. L. Shew, H. Yang, T. Petermann, R. Roy, and D. Plenz, Neuronal avalanches imply maximum dynamic range in cortical networks at criticality, J. Neurosci. 29, 15595 (2009).

[7] M. Girardi-Schappo, G. S. Bortolotto, J. J. Gonsalves, L. T. Pinto, and M. H. R. Tragtenberg, Griffiths phase and long-range correlations in a biologically motivated visual cortex model, Sci. Rep. 6, 29561 (2016).

[8] T. S. Mosqueiro and L. P. Maia, Optimal channel efficiency in a sensory network, Phys. Rev. E 88, 012712 (2013).

[9] A. Levina, J. Michael Herrmann, and T. Geisel, Dynamical synapses causing self-organized criticality in neural networks, Nat. Phys. 3, 857 (2007).
[10] J. A. Bonachela, S. deFranciscis, J. J. Torres, and M. A. Muñoz, Self-organization without conservation: Are neuronal avalanches generically critical? J. Stat. Mech. (2010) P02015.

[11] J. Peng and J. M. Beggs, Attaining and maintaining criticality in a neuronal network model, Physica A 392, 1611 (2013).

[12] A. A. Costa, M. Copelli, and O. Kinouchi, Can dynamical synapses produce true self-organized criticality? J. Stat. Mech. (2015) P06004.

[13] J. G. F. Campos, A. A. Costa, M. Copelli, and O. Kinouchi, Correlations induced by depressing synapses in critically selforganized networks with quenched dynamics, Phys. Rev. E 95, 042303 (2017).

[14] L. Brochini, A. A. Costa, M. Abadi, A. C. Roque, J. Stolfi, and $\mathrm{O}$. Kinouchi, Phase transitions and self-organized criticality in networks of stochastic spiking neurons, Sci. Rep. 6, 35831 (2016).

[15] A. A. Costa, L. Brochini, and O. Kinouchi, Self-organized supercriticality and oscillations in networks of stochastic spiking neurons, Entropy 19, 399 (2017).

[16] O. Kinouchi, L. Brochini, A. A. Costa, J. G. F. Campos, and M. Copelli, Stochastic oscillations and dragon king avalanches in self-organized quasi-critical systems, Sci. Rep. 9, 3874 (2019). 
[17] J. A. Bonachela and M. A. Muñoz, Self-organization without conservation: true or just apparent scale-invariance? J. Stat. Mech. (2009) P09009.

[18] N. A. Lambert and W. A. Wilson, Temporally distinct mechanisms of use-dependent depression at inhibitory synapses in the rat hippocampus in vitro, J Neurophysiol. 72, 121 (1994).

[19] C. W. G. Clifford, M. A. Webster, G. B. Stanley, A. A. Stocker, A. Kohn, T. O. Sharpee, and O. Schwartz, Visual adaptation: Neural, psychological and computational aspects, Vision Res. 47, 3125 (2007).

[20] Zhengyu Ma, G. G. Turrigiano, R. Wessel, and K. B. Hengen, Cortical circuit dynamics are homeostatically tuned to criticality in vivo, Neuron 104, 655, (2019).

[21] S.-S. Poil, R. Hardstone, H. D. Mansvelder, and K. LinkenkaerHansen, Critical-state dynamics of avalanches and oscillations jointly emerge from balanced excitation/inhibition in neuronal networks, J. Neurosci. 32, 9817 (2012).

[22] F. Lombardi, H. J. Herrmann, C. Perrone-Capano, D. Plenz, and L. de Arcangelis, Balance between Excitation and Inhibition Controls the Temporal Organization of Neuronal Avalanches, Phys. Rev. Lett. 108, 228703 (2012).

[23] F. Lombardi, H. J. Herrmann, and L. deArcangelis, Balance of excitation and inhibition determines $1 /$ f power spectrum in neuronal networks, Chaos 27, 047402 (2017).

[24] L. DallaPorta and M. Copelli, Modeling neuronal avalanches and long-range temporal correlations at the emergence of collective oscillations: Continuously varying exponents mimic M/EEG results, PLoS Comput. Biol. 15, e1006924 (2019).

[25] N. Brunel, Dynamics of sparsely connected networks of excitatory and inhibitory spiking neurons, J. Comput. Neurosci. 8, 183 (2000).

[26] P. Somogyi, G. Tamás, R. Lujan, and E. H. Buhl, Salient features of synaptic organisation in the cerebral cortex, Brain Res. Rev. 26, 113 (1998).

[27] W. Gerstner and J. Leo van Hemmen, Associative memory in a network of 'spiking' neurons, Network: Comput. Neural Syst. 3, 139 (1992).
[28] A. Galves and E. Löcherbach, Infinite systems of interacting chains with memory of variable length - a stochastic model for biological neural nets, J. Stat. Phys. 151, 896 (2013).

[29] J. Benda and A. V. M. Herz, A universal model for spikefrequency adaptation, Neural Comput. 15, 2523 (2003).

[30] M. A. Muñoz, R. Dickman, A. Vespignani, and S. Zapperi, Avalanche and spreading exponents in systems with absorbing states, Phys. Rev. E 59, 6175 (1999).

[31] S. Lübeck, Universal scaling behavior of non-equilibrium phase transitions, Int. J. Mod. Phys. B 18, 3977 (2004).

[32] M. Girardi-Schappo and M. H. R. Tragtenberg, Comment on Convergence towards asymptotic state in 1-D mappings: A scaling investigation, Phys. Lett. A 383, 126031 (2019).

[33] D. R. Chialvo, Emergent complex neural dynamics, Nat. Phys. 6, 744 (2010).

[34] M. A. Muñoz, Colloquium: Criticality and dynamical scaling in living systems, Rev. Mod. Phys. 90, 031001 (2018).

[35] C. vanVreeswijk and H. Sompolinsky, Chaos in neuronal networks with balanced excitatory and inhibitory activity, Science 274, 1724 (1996).

[36] G. S. Bortolotto, M. Girardi-Schappo, J. J. Gonsalves, L. T. Pinto, and M. H. R. Tragtenberg, Information processing occurs via critical avalanches in a model of the primary visual cortex, J. Phys. Conf. Ser. 686, 012008 (2016).

[37] M. Girardi-Schappo and M. H. R. Tragtenberg, Measuring neuronal avalanches in disordered systems with absorbing states, Phys. Rev. E 97, 042415 (2018).

[38] J. M. Beggs and D. Plenz, Neuronal avalanches in neocortical circuits, J. Neurosci. 23, 11167 (2003).

[39] Chong-Yang Wang, Zhi-Xi Wu, and Michael Z. Q. Chen, Approximate-master-equation approach for the KinouchiCopelli neural model on networks, Phys. Rev. E 95, 012310 (2017).

[40] A. Renart, J. delaRocha, P. Bartho, L. Hollender, N. Parga, A. Reyes, and K. D. Harris, The asynchronous state in cortical circuits, Science 327, 587 (2010).

[41] Ł. Kuśmierz, S. Ogawa, and T. Toyoizumi, Edge of chaos and scale-free avalanches in neural networks with heavy-tailed synaptic disorder, arXiv:1910.05780 [physics.bio-ph]. 